\title{
Approaches of Collaborative Cloud Process Service: Constructing Service-Oriented Architecture
}

\author{
Chen Wang ${ }^{1}$, Wu Zhao ${ }^{1 *},{ }^{1}$ Jie Wang ${ }^{1}$ and Ling Chen ${ }^{2}$ \\ ${ }^{1}$ School of Manufacturing Science and Engineering, \\ Sichuan University, \\ Cheng Du 610065 China; \\ ${ }^{2}$ Division of Production and Materials Engineering, \\ Lund University, Lund, 999027, Sweden \\ mr.wangchen@foxmail.com
}

\begin{abstract}
Process service is an important segment of cloud manufacturing ecosystem, in which virtualized process resources are furnished as consumable services. In this paper, a service-oriented approach cloud process service is proposed to achieve a collaborative processing environment for distributed participants. In order to implement the cloud process service, it's important to identify the existing process resources and capabilities, then virtualize them as processing services. In the first place, basic features of process service are summarized, and a standardized business model and process knowledge base are built to describe concept, cloud service and relevant features. Besides, a seven layer structure of cloud platform is proposed with a virtualization methodology to describe process service, source provider and process service requests. In the end, a case is presented to demonstrate the ability of integrating cloud process services.
\end{abstract}

Keywords: cloud manufacturing, process service, knowledge base, Service-oriented manufacturing

\section{Introduction}

The Internet connects the world and brings the opportunities to share resource and knowledge in a collective way. On the other hand, manufacturing enterprises around the world are struggling to seek more effective manufacturing strategies. The concept of cloud computing was introduced into manufacturing, to create the concept of cloud manufacturing. Cloud manufacturing is a customer-centric manufacturing model that exploits on-demand access to a shared collection of diversified and distributed manufacturing resources to form temporary, reconfigurable production capacities which enhance efficiency, reduce costs [1]. In 2010 the European Commission launched a research project on cloud-based manufacturing with the purpose of integrating distributed and virtualized production resource based on networks and providing manufacturing enterprises with the cloud-based manufacturing service [2].

The cloud-based process service as well as other cloud services (such as design, simulation services, etc.) are important part in the concept of cloud manufacturing. With the support of relevant domain knowledge base, the relative virtualized process resources and manufacturing ability can be integrated in a platform to form process cloud services [3]. The implementation of cloud manufacturing service platform is a systematic project, there are still lots of technical issues need to be solved. The related process resource, capability and knowledge etc., need to be well described, componentized, virtualized, in

$\mathrm{Wu} \mathrm{Zhao}^{1 *}$, is the corresponding author. 
order to be integrated in process service cloud. Lots of researches on cloud manufacturing has made recently both on resource virtualization and system architecture. Wu and Yang [4] proposed an approach on resource modelling to describe the manufacturing resources in a cloud environment. Hu et al. [5] proposed a method about the classification of virtual manufacturing resources in cloud manufacturing. Fan and Xiao [6] proposed an architecture on resource integration based on a federation principle.

However, the researches above are mainly concerning the whole structure of cloud manufacturing, the research on solution for process cloud service is insufficient. There is a need for further research in the field of process cloud service. This paper will analyze the some key technologies and implementation method of the process cloud service combining the characteristics of process service. The process cloud service are discussed followed by the presentations of relevant researches that help the implementation of a process cloud service in the first half of this paper. Besides, a model of process service cloud platform is proposed in the second half of this paper.

\section{Process Cloud Service}

Process resources are virtualized and encapsulated into Process Cloud Services [7]. Shown as Fig. 1, the Process Cloud Services environment is enabled by users, resource providers, and cloud plant operators with the support of knowledge base. The operator is responsible for establishing operation mode and operating service platform; Resource provider provides cloud-based process services to the demander based on its own process resources and manufacturing capacity; User can obtain cloud process service by means of pay-as-you-go. For example, $\mathrm{CNC}$ machining services are represented into process service cloud which is a constituent part of manufacturing cloud. User can search this kind of service in the manufacturing cloud and pay related fees to buy related service.

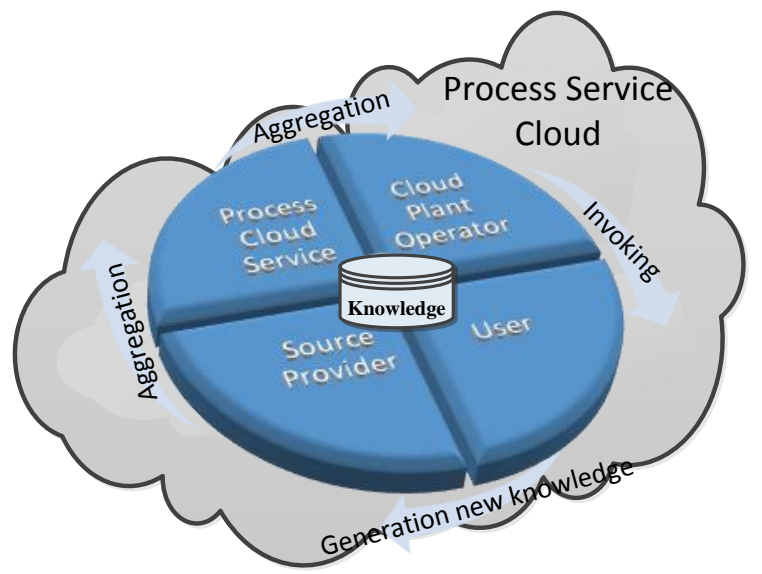

Figure 1. Cloud Manufacturing Abstract

In the concept of cloud manufacturing, process resources usually have the characteristics of dispersed, autonomy, heterogeneity and dynamic [8], the process service needs to solve several common problems, such as the perception and access of virtual resources, encapsulation of service, etc. Therefore, in addition to the common problems of cloud services, full consideration on the special requirements of process field is needed. Specific to the process cloud services, virtual resources and technical process need to be integrated into cloud manufacturing environment, including a series of necessary work, such as process design, NC programming and simulation and so on. 
For establishing process cloud services, both hardware resources and soft resources need to be integrated [9]. Hardware resources which represented by numerical control machine tool need to be described by uniform data model or specification. The cloud service is also closely related to process, technology, and skills which are determined by knowledge and software. There are different ways of the expression and storage of process knowledge [10], such as forms of technological knowledge handbook, specification of machine, as well as the code of NC machining tool and so on. Therefore, reasonable knowledge modeling of process capability is also very important.

\section{The Virtualization of Process Resource}

The distribution of integrated resources and the integration of distributed resources are the key features of cloud process service. Resource virtualization is a necessary condition for the servitization of manufacturing capability [11]. Distributed process resources and process capabilities need to be integrated to form a resource pool and assigned to user depending on requirement [12]. On the other hand, for purpose of facilitating rapid scalability, it's crucial to formally represent the process resources.

\subsection{The Description of Cloud Process Resources}

For better description and presentation of cloud process service, provider and user's requests, it's necessary to build an efficient data models with robust modelling language. EXPRESS is a kind of standard data modeling language which is used for product data, and it is also formalized in the ISO Standard for the Exchange of Product model STEP. The method of describe the cloud process resource based on EXPRESS-G can be summarized as follows.

1. Classification

The resource type can be divided into and software resources (S_resource) and hardwarresource (H_resource). Then each of them can be further classified into different groups.

2. Description

Resources can be described by the classification of basic properties, performance and function attributes, transaction attribute and evaluation attribute, each of these attributes may continue to be subdivided [13]. For example, a knowledge model of a $\mathrm{CNC}$ milling machine can be obtained via describing it from the above five aspects.

3. Instantiation

According to the above knowledge model and EXPRESS language, instantiation of cloud process resources can be obtained through giving assignments to parameters of attribute.

The participator models can be expressed in EXPRESS which provides the portability with standard data models, these enterprises which provide cloud process services are defined as Cloud_Process_Service_Provider. The elements in process service cloud such as distributed process resources (e.g., equipments, materials, software, knowledge, and skills) and process capabilities (e.g., process design capability, production capability, experimentation capability, and management capability) can also be expressed as Fig. 2. In this way, manufacturing enterprises can be described by provider profiles and service properties in the cloud environment. And the attributes of process resource including both Hard_Resouce and Soft_Resource are represented. 


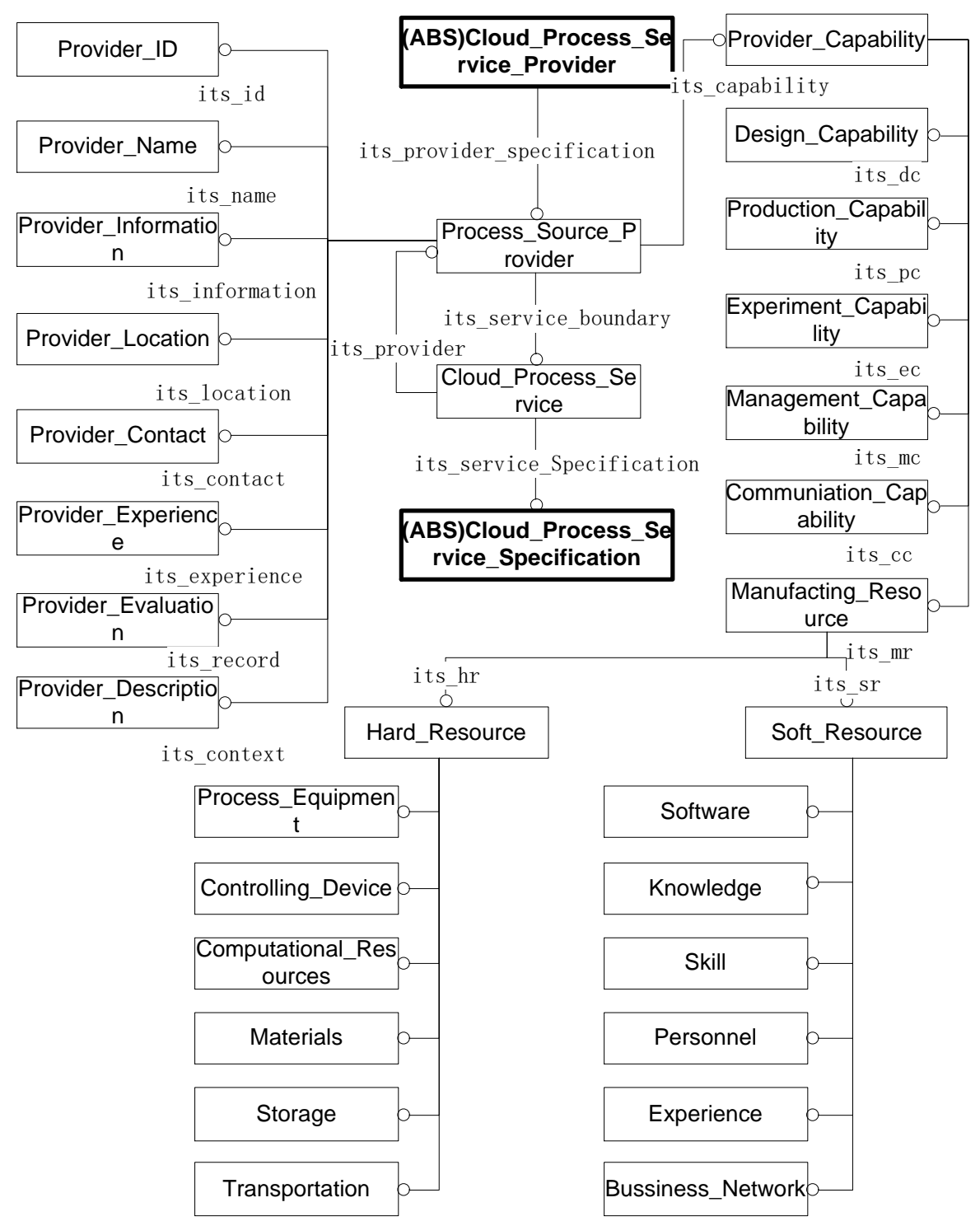

Figure 2. The Cloud Process Participator Model

Cloud platform need to provide enterprises with detailed information on collaborative process planning, assigning task and execution trace to realize collaborative management. Besides, to describe the second category of enterprise attributes further, the recognition of the specific cloud process service is modelled via Entity Cloud_Process_Service_Template, shown as Figure 3, This entity gives the explicit model of the value which is used to accomplish process service. 


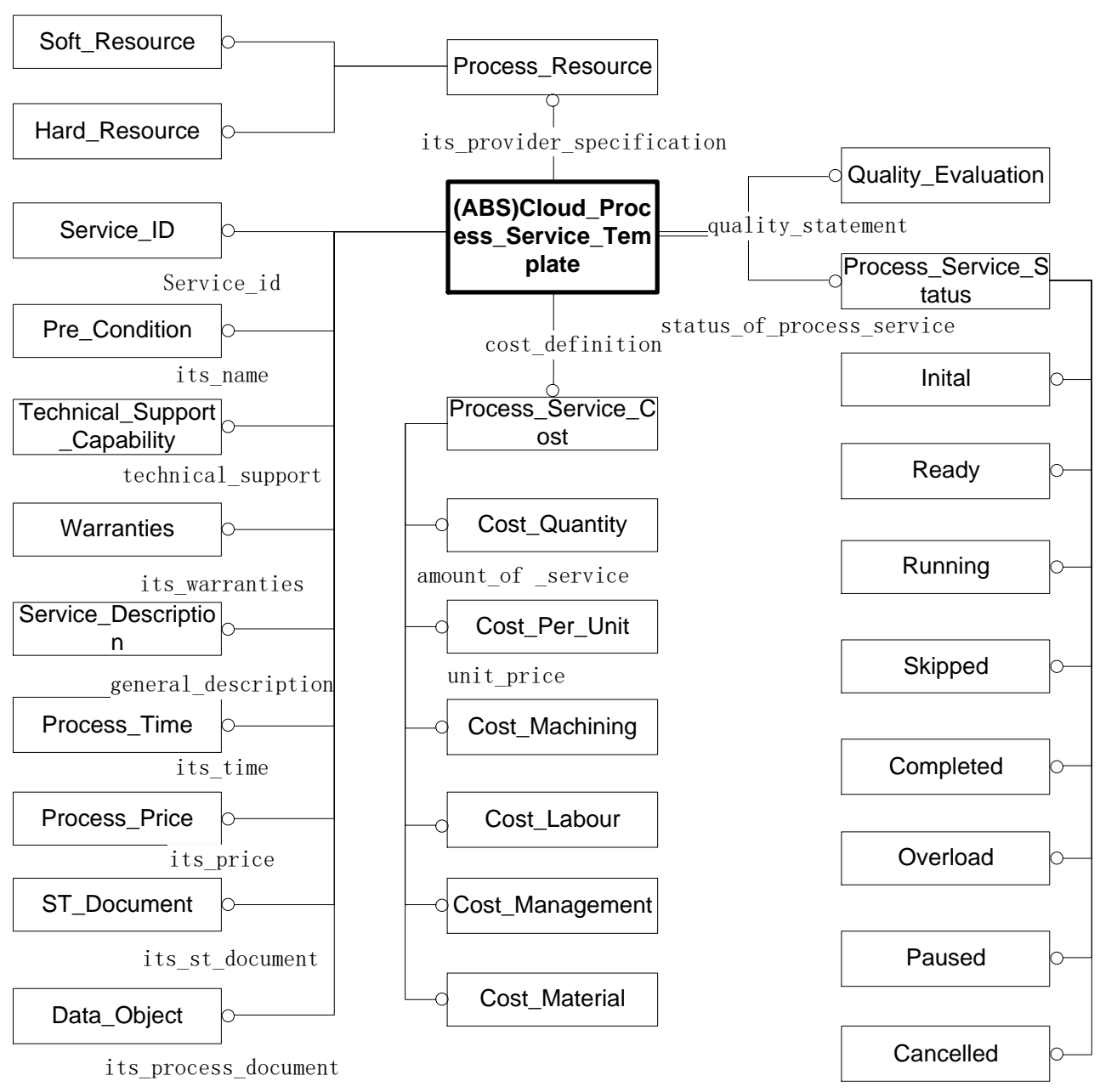

Figure 3. The Cloud Process Service Templates Model

Cloud_Service_Request model is also built to describe the request of a cloud process service, shown as Figure 4, Cloud_Process_Service_Request is a bridge between process demand and cloud process service, this model offers a standardized methods for the users' request. In practice, the service description is presented as a structured statement and transmitted to the knowledge base which can give suggestion based on the resource pool and mapping rules. 


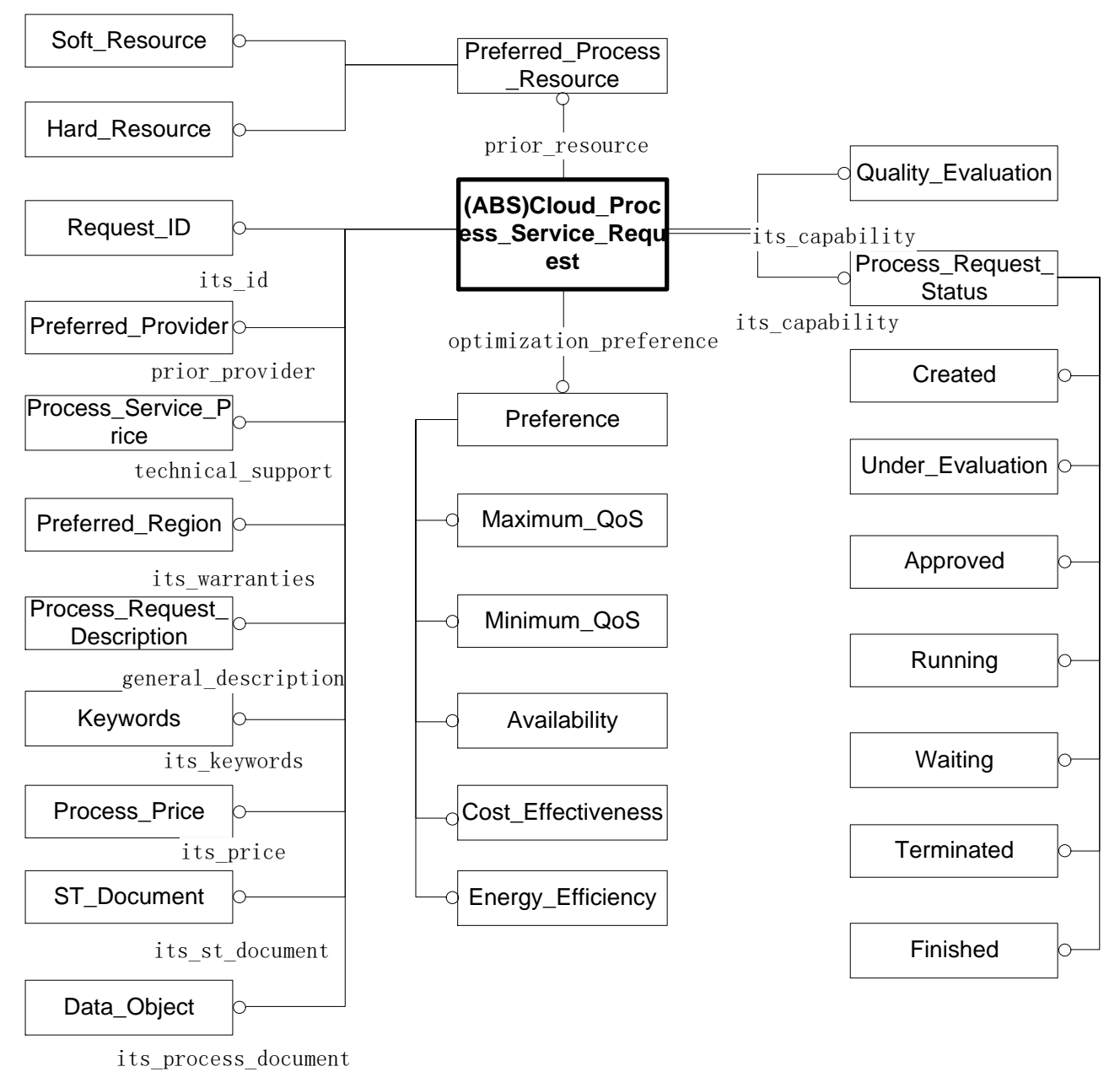

Figure 4. The Cloud Process Service Request Model

\subsection{The Process Knowledge Base}

Process involves lot of information and knowledge, such as geometry information of work piece [14], NC system and programming language, the types of machining tool, cutting parameters and optimization methods, etc. These knowledge acts as a core supporting role in cloud manufacturing system. To implement loud process service, process knowledge need to be well expressed and organized to build process knowledge base.

In order to meet the demand of cloud process service, this paper built a process knowledge base model based on ontology technology to describe relative concepts, relations, rules, etc. As shown in Fig 5, structure of process knowledge ontology mainly includes the rule layer, concept layer and domain layer. The concept layer describes the related conceptions, the relationship of attributes and constraints; Domain layer is mainly used to store instances corresponding to the conceptions in concept layer; Rule layer is used to collect related rules.

The first priority of constructing concept layer is to classify the relevant subject knowledge, and list every concepts and attributes within the scope of subject, then organize it by ontology language to resulting in a set of ontology concepts. As shown in Fig 5, the related process concepts are divided into process characteristics ontology, process technology ontology, cutting tool ontology and NC system Ontology, etc. with the help of Web Ontology Language. (Fig 5 only shows the part of conception set and its relationship). 
Domain layer is mainly used to store the instance corresponding to concept layer, all instances in domain layer has property values. For example, the concept of hole processing is defined in concept layer, there will be a specific instance indicated as ontology individual to describe the information of hole processing in the domain layer.

The rule layer which is part of knowledge base contains the racer and domain rules, and can be updated and expanded, racer enables the knowledge base to reasoning a solution based on domain rules. This layer is the foundation of realizing intelligent decision, such as process planning and selection of cutting tool. Semantic Web Rule Language is applied to describe the inference rules. For example, the rule for automatic selecting cutting tool is shown as below:

DrillingCuttingTool $\left(? \mathrm{r}_{1}\right) \wedge$ hasToolRadius $\left(? \mathrm{r}_{1}, ? \mathrm{~m}\right) \wedge$ HoleFeature $\left(? \mathrm{r}_{2}\right) \wedge$ has Diameter $\left(? \mathrm{r}_{2}, ? \mathrm{n}\right) \wedge$ swrlb: Equal(?m,?n) $\rightarrow$ isSelected(? $\mathrm{r}_{1}$, ture $)$

The rules means that if the drilling diameter $r$ has the same value as the characteristics of hole $\mathrm{r} 2$, the drilling cutter can be selected to perform this task.

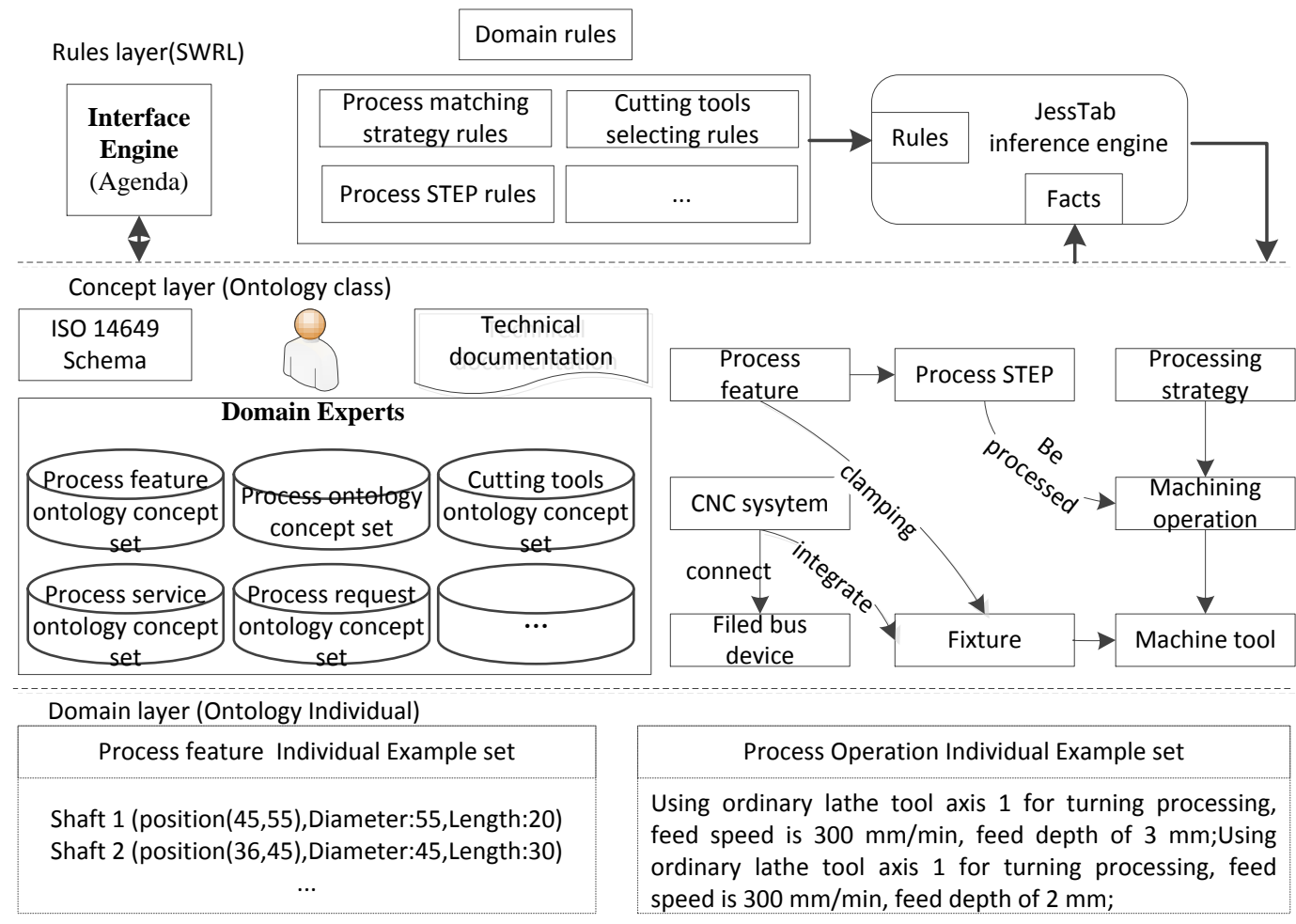

Figure 5 .The Process Knowledge Base Based on Ontology

\section{Cloud Process Service Platform}

The cloud process services system integrates process resources, information, and management service together, can provide more professional and stable service [15]. It can not only effectively use process resources to provide service for user scattered in different locations, but also can effectively integrate distributed manufacturing capacity to form a composite service for the whole machining process. 


\subsection{The Logic of Process Service}

In order to describe the workflow accurately, it is necessary to understand the procedure from raw material to finished product. As shown in Fig 6, the cloud process service can be roughly divided into two stages of matching and monitoring from the perspective of service management. Process request form user will be analyzed to determine the technological characteristics, and decomposed into sub-tasks according to the resource existing in cloud. Then these sub-tasks match process resources according to rules and related constraints. The purpose of this stage is to realize the mapping of process tasks and manufacturing resources/manufacturing capability. After that, the process service is realized by the implementation of scheduling, optimization, process planning, programming and processing, etc. The cloud process service is composed by five service phases. According to the sequence of different routes, the cloud process service procedure can be presented in the flowchart as below.

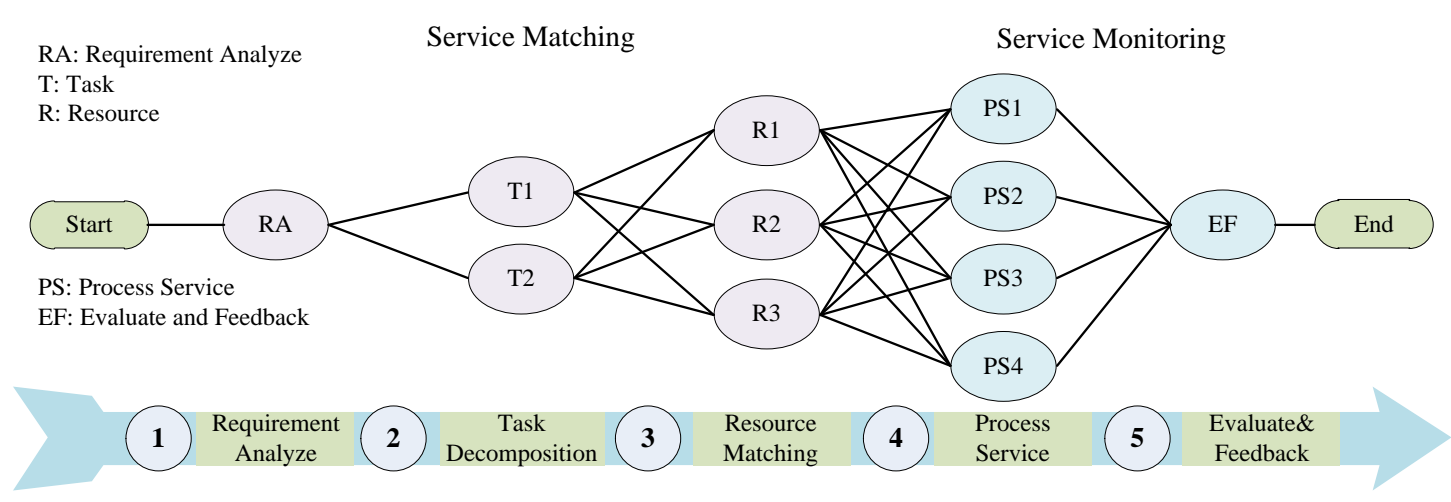

Figure 6. Flowchart of Cloud Process Service

\subsection{System Architecture}

The cloud process service platform is an independent cloud service system, can be seen as a subsystem of the entire cloud manufacturing system which focuses on the whole life cycle manufacturing [16]. The cloud process service platform mainly supports the services in the field of manufacturing process, and pays more attention to the application integration and the management of knowledge than the general cloud service platform. In order to ensure the overall production completing on schedule with high quality, the processing progress, quality and manufacturing process of external resources must be closely integrated. At the same time, platform integrates with the ERP system to realize the exchange of information between cloud manufacturing platform and enterprise internal management system. Participators of cloud platform include resource provider, demander and platform operator. The demanders, most of them are small and mediumsized manufacturing enterprises, will collaborate with external resources at the manufacturing process level.

The architecture of process service cloud platform is shown as Figure 7, which including the user layer, the core function layer, service model layer, application integration layer, virtual resources layer, supporting layer and running environment layer.

1) The user layer. User layer provide unified interface for process service demander and provider, user can login the platform via a web browser around the world, publish or request process service, participate in the whole process of cloud manufacturing. 
2) The core function layer. This layer offer users the main functions of cloud process service, based on various types of virtualization resources. These functions include resource publishing, request publishing, service registry, matching management, transaction management, service management, credit evaluation, knowledge accumulation and user management, etc.

3) Service model layer. The main function of this layer is to realize the cooperation of different participators.

4) Application integration layer. Application integration layer offers the function of resources transformation and information sharing, and supports the accession to virtualized resources. It also provides a data interface, use STEP-NC (Standard for the Exchange of Product model data - compliant Numerical Control data interface) to realize data exchange and interoperability. In addition, this layer not only communicates with CAD/CAM software, but also supports knowledge base.

5) Virtual resources layer. It covers various types of process resources which can be divided into hardware and software resources depending on their properties.

6) Supporting layer. It is the base layer of platform, mainly providing cloud storage platform, network connecting, database and server based support.

7) Running environment layer. Lasting and stable operation of the platform is guaranteed by the running environment layer which including agreements, security, operational standards and monitoring environment, etc.

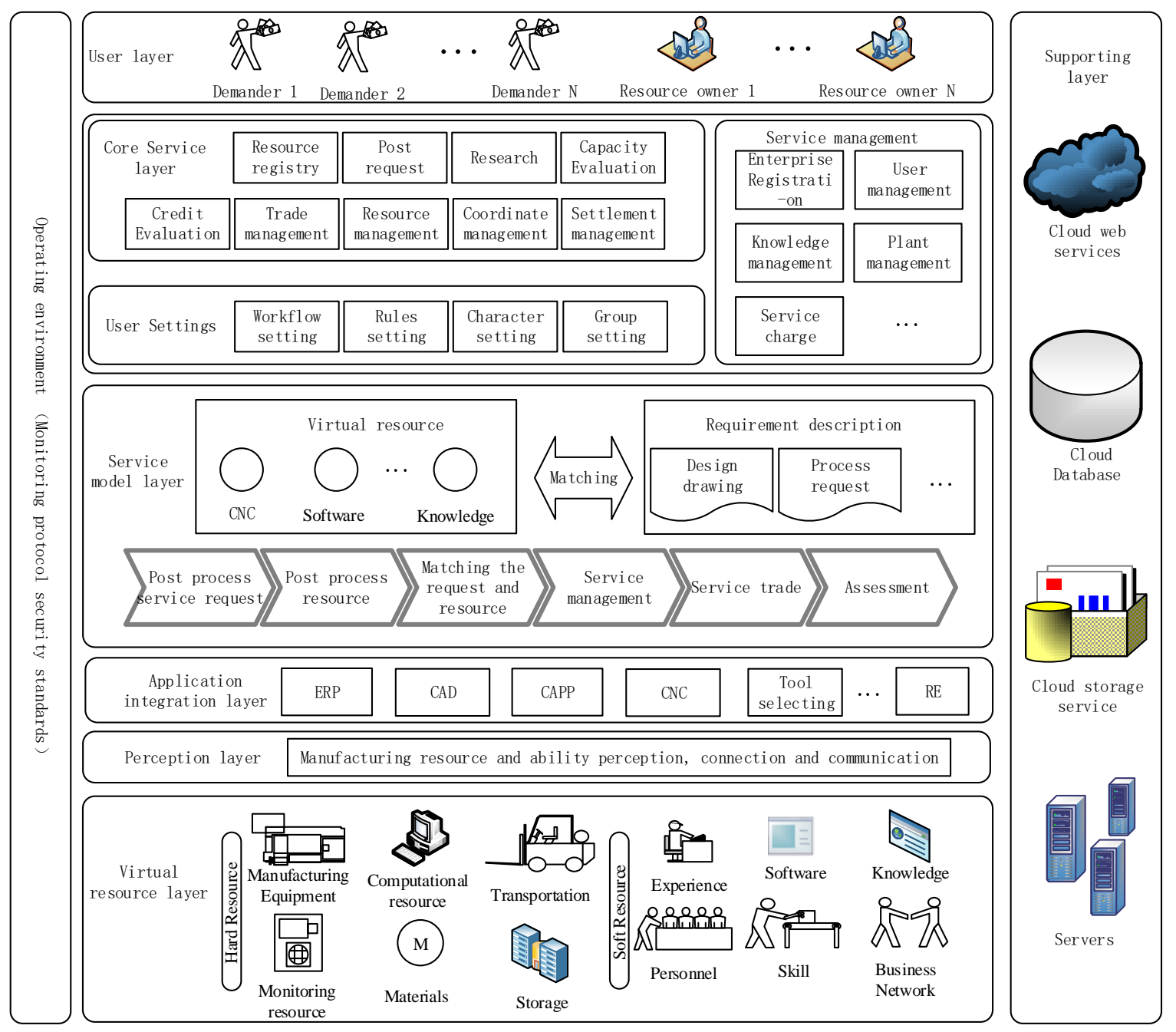

Figure 7. The Architecture of Process Service Cloud Platform 


\section{Case Studies}

The process service cloud platform offers a possibility to break the barrier between organizations and geography. Process requirement can be decomposed into several subtasks to be completed by service providers around the world efficiently and economically.

To evaluate the concept of cloud process services, a case of Platform as a Service $(\mathrm{PaaS})$ is presented as Fig. 8 to demonstrate the ability of integrating cloud process services. User can login the system dashboard and upload service requests by filling out process requirements and upload relevant documents on request-upload page. For example, the user requests a process planning service of generating detailed information. The process requirements will be stored in XML format. After the service request is received, the system will analyze the process request and search authorized process resources for user by cloud management engine according to the rules stored in knowledge base. System retrieves detailed information about every service provider including process capabilities, credibility, etc. Then, certain process resources are returned to system after searching all the database about service resource. User also can view progress of the process service.

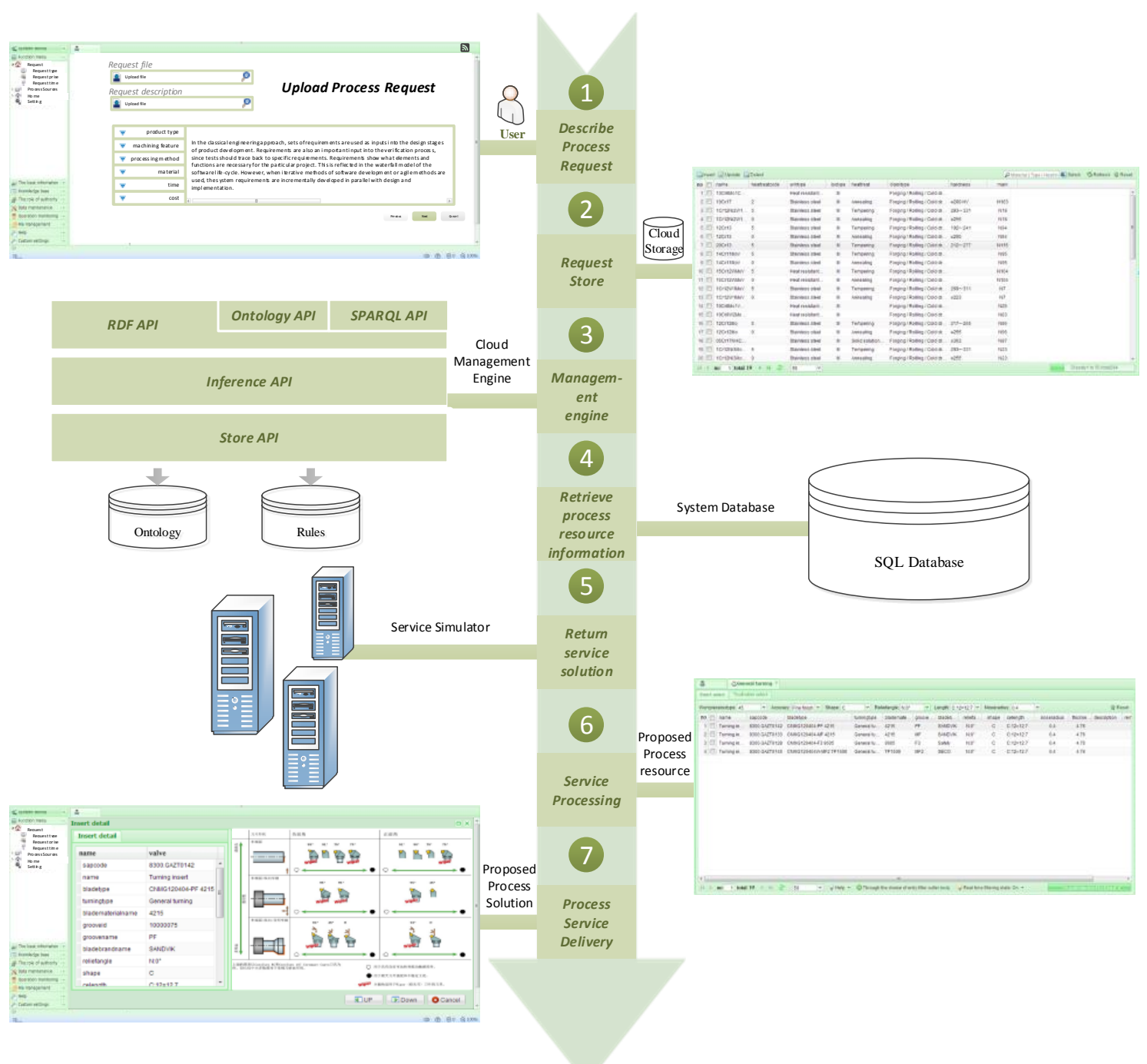

Figure 8. The Flow Path of Cloud Process Service Platform 


\section{Conclusions}

In this paper, the prototype system of cloud process service platform is proposed to achieve a collaborative processing environment for all participants including process demander, process service provider and platform operator. In order to implement the cloud process service, it's important to identify the existing process resources and capabilities, then virtualize them as processing services. Therefore, a seven-layer structure of cloud platform is proposed with a virtualization methodology to describe process service, source provider and process service requests. A case is presented to demonstrate the ability of integrating cloud process services. Besides, additional applications and software can be adopted in the cloud platform. This cloud process service platform connects distributed process resources to provide services for user distributed around the world. In the future, more powerful knowledge base is needed to describe numerous manufacturing process type.

\section{Acknowledgement}

This work was supported in part by NSFC (National Natural Science Foundation of China) under Grant No.51175357, National High-tech Research and Development Projects (863) under Grant NO.2013AA040606 and Sichuan Province Science and technology support program under Grant No. 2014GZ0114. Gratitude is also extended to the reviewers and the Editor for their valuable comments.

\section{Conflict of Interests}

The authors declare that there is no conflict of interests regarding the publication of this paper.

\section{Reference}

[1] D. Wu, MJ Greer, DW Rosen, D Schaefer, "Cloud manufacturing: drivers, current status, and future trends." In: Proceedings of the ASME 2013 international manufacturing science and engineering conference (MSEC13), Paper Number:MSEC2013-1106, Madison, Wisconsin, US. (2013).

[2] W. Terkaj, , \& M. Urgo,. "Virtual Factory Data Model to support Performance Evaluation of Production Systems". In Proceedings of OSEMA 2012 workshop, 7th international conference on formal ontology in information systems, Graz, Austria, July (2012), pp. 24-27.

[3] C. Yin, B. Q Huang, F. Liu, , Wen, L. J., Wang, Z. K., Li, X. D., \& Liu, X. H. Common key technology system of cloud manufacturing service platform for small and medium enterprises. Computer Integrated Manufacturing Systems, vol. 17, no. 3, (2011), p. 495-503.

[4] L. Wu, ., \& C. Yang. "A solution of manufacturing resources sharing in cloud computing environment. In Cooperative Design”, Visualization, and Engineering, (2010). (pp. 247-252).

[5] Hu, C. S., Xu, C. D., Cao, X. B., \& Fu, J. C. (2012). Study of classification and modeling of virtual resources in Cloud manufacturing. Applied Mechanics and Materials, 121, 2274-2280.

[6] W. H Fan, \& T. Y Xiao, "Integrated architecture of cloud manufacturing based on federation mode." Computer Integrated Manufacturing Systems, vol .17, no. 3, pp. 469-476.

[7] U .Rauschecker, M Stöhr,., \& D. Schel,’Requirements and Concept for a Manufacturing Service Management and Execution Platform for Customizable Products" In ASME 2013 International Manufacturing Science and Engineering Conference collocated with the 41st North American Manufacturing Research Conference (pp. V002T02A001-V002T02A001). American Society of Mechanical Engineers. June (2013).

[8] S. Yin, C. Yin , F Liu., \& Li, X. B. "Outsourcing resources integration service mode and semantic description in cloud manufacturing environment" Computer Integrated Manufacturing Systems, vol. 17, no.3, (2011), pp. 525-532.

[9] H. K Lin, \& Harding, J. A. "A manufacturing system engineering ontology model on the semantic web for inter-enterprise collaboration”. Computers in Industry, vol .58, no. 5, (2007). pp 428-437.

[10] F. Tao, L. Zhang, V. C Venkatesh, Y. Luo, \&Y. Cheng, "Cloud manufacturing: a computing and service-oriented manufacturing model". Proceedings of the Institution of Mechanical Engineers, Part B: Journal of Engineering Manufacture, 0954405411405575, (2011). 
[11] S. T Newman,., \& A Nassehi,. (2007). "Universal manufacturing platform for CNC machining”. CIRP Annals-Manufacturing Technology, vol. 56, no. 1, pp. 459-462.

[12] F. Tao, Y. Cheng, L. Zhang, D. Zhao . "Utility modelling, equilibrium, and coordination of resource service transaction in service-oriented manufacturing system" Proceedings of the Institution of Mechanical Engineers, Part B: Journal of Engineering Manufacture , vol. 226, no. 6, (2012); pp. 1099-117.

[13] M . Sensoy, \& P. Yolum, "Ontology-based service representation and selection. Knowledge and Data Engineering", IEEE Transactions on, vol. 19, no. 8, (2007), pp. 1102-1115.

[14] P. Vichare, A. Nassehi., S. Kumar, \& Newman, S. T. "A Unified Manufacturing Resource Model for representing CNC machining systems", Robotics and Computer-Integrated Manufacturing, vol. 25 , no. 6 , pp. 999-1007.

[15] M. Cai, W. Y Zhang, \& K. Zhang,"ManuHub: a semantic web system for ontology-based service management in distributed manufacturing environments". Systems, Man and Cybernetics, Part A: Systems and Humans, IEEE Transactions on, vol. 41, no. 3, (2011), pp. 574-582.

[16] D. Wu, J. L Thames, D. Rosen,. W., \& Schaefer, D, "Enhancing the Product Realization Process With Cloud-Based Design and Manufacturing Systems" Journal of Computing and Information Science in Engineering, vol. 13, no. 4, (2013). 041004.
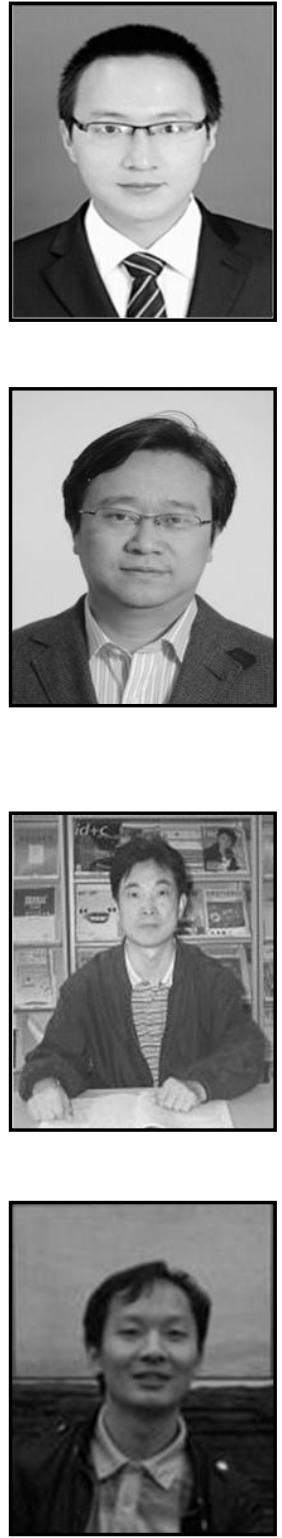

\section{Authors}

Chen Wang, he is studying for a doctor's degree in Mechanical Manufacture and Automation at Sichuan University of China. His research interests are in the areas of innovative design and knowledge base including the mathematical methods and models for complex systems. He has published research articles in several international journals.

WU Zhao, he received the $\mathrm{PhD}$ degree in Mechanical Manufacture and Automation at Sichuan University of China. He was one of the Innovation method and the design key laboratory of sichuan province. His research interests are in the areas of innovative design and knowledge base including the mathematical methods and models for complex systems. He has published research articles in reputed international journals. He is referee and editor of several reputed international journals.

Jie Wang, he was born in February 1964, doctoral supervisor, institute of manufacturing vice president and director of engineering training center. The main research direction: computer aided design and manufacture (CAD/CAM); Computer integrated manufacturing system (CIMS).

Ling Chen, he was born in February 1986, studying for a doctor's degree in Division of Production and Materials Engineering, Lund University of Sweden. The main research direction: computer aided design and manufacture (CAD/CAM); Computer integrated manufacturing system (CIMS). 